\title{
Heating of the Beurling operator: Sufficient conditions for the two-weight case
}

by

S. Petermichl (Bordeaux) and J. Wittwer (Salt Lake City, UT)

\begin{abstract}
We establish sufficient conditions on the two weights $w$ and $v$ so that the Beurling-Ahlfors transform acts continuously from $L^{2}\left(w^{-1}\right)$ to $L^{2}(v)$. Our conditions are simple estimates involving heat extensions and Green's potentials of the weights.
\end{abstract}

\section{Notation.}

- $C$ a constant not depending on any functions, other than the weights. $C$ will not necessarily denote the same constant at each occurrence.

- $x:=\left(x_{1}, x_{2}\right), \partial_{1} f(x):=\partial f / \partial x_{1}$.

- $\Phi(x, t)=\Phi_{t}(x):=(4 \pi t)^{-1} e^{-\|x\|^{2} / 4 t}$ the heat kernel on the plane.

- $\widetilde{f}(x, t)$ the heat extension of a function $f(x)$ in the plane:

$$
\widetilde{f}(x, t):=\iint f(x-y) \Phi_{t}(y) d A(y) .
$$

- $\mathcal{G}(f)(x, t)$ the Green's potential of a function $f(x, t)$ :

$$
\mathcal{G}(f)(x, t):=\int_{0}^{t} \int_{\mathbb{R}^{2}} \Phi(x-y, t-s) f(y, s) d A(y) d s .
$$

- $C\left(R, R^{2}\right)=B(0, R) \times\left(0, R^{2}\right)$ a cylinder in $\mathbb{R}_{+}^{3}$.

- $\bar{C}^{\delta}\left(R, R^{2}\right)=\bar{B}(0, R-\delta) \times\left[\delta,(R-\delta)^{2}\right]$ a slightly smaller compact cylinder.

- $R_{1}, R_{2}$ the Riesz transforms in $\mathbb{R}^{2}, R_{i}(f)=f * K_{i}, K_{i}(x)=c_{n} x_{i} /|x|^{3}$.

1. Introduction. The goal of this paper is to present a set of conditions on the weights $w$ and $v$ so that the Beurling operator from $L^{2}\left(w^{-1}\right) \rightarrow L^{2}(v)$ is bounded. The Beurling operator is a two-dimensional analogue of the

2000 Mathematics Subject Classification: Primary 30Exx.

Key words and phrases: Bellman functions, weights, Beurling operator.

Research of S. Petermichl was supported by NSF grant DMS-0300255.

Research of J. Wittwer was supported by NSF grant DMS-0507993. 
Hilbert transform and plays an important role in the study of differential equations.

Two-weight estimates for the Beurling operator come up naturally in various areas of analysis, perturbation theory and probability theory. They are connected to a well-known homotopy problem for chord arc curves [2].

It has been understood for a long time that dyadic operators, such as martingale transforms, are an excellent model for singular integral operators like the Hilbert transform, the Beurling operator and the like. The method of Bellman functions is well suited to tackling problems that find their origins in dyadic estimates. This method reduces dyadic sum estimates to the existence of a function with certain restrictions on its domain, range and some differential inequalities. It probably first appeared in harmonic analysis in the works by Burkholder on martingale inequalities in [4]-[6]. It seems though that it had not been understood how universal it was until it was reinvented by Nazarov, Treil and Volberg in their work concerning two-weight questions for Haar multipliers in [11]; it was then extensively developed in [9], and was a major tool in [8], [13], [14], [19], [16], [17], [15], [12], [10].

Many of these involved surprising mutations of the original. For example, in [16] we used some of the ideas and techniques from [19] to prove a sharp weighted bound for the Hilbert transform. This required the extension of the methods of [11] and [19] to the continuous case with harmonic extensions, relying on a Littlewood-Paley identity involving the Hilbert transform.

In this paper, rather than using harmonic extensions of the weights, we will use heat extensions, which make working with the Beurling operator particularly nice. In [15], sharp results for the one-weight estimate of the Beurling operator were obtained by using a heat extension of the Muckenhoupt $A_{2}$ condition. This result in turn solved a long standing problem in regularity theory for Beltrami equations (see [1] and [3]).

If one considers the case of one weight, a condition involving heat extensions of the weights is necessary and sufficient for boundedness. Moreover, the condition involving the heat extension is similar to the classical Muckenhoupt condition in the sense that the ratios of the classical and heat characteristics are bounded. In this paper we also frame the conditions on the two weights in terms of their heat extensions, and in terms of potentials involving the Green's function of the heat equation. We establish linear and bilinear embedding theorems for this situation. Our conditions on the weights are the appropriate conditions for the embedding to hold.

2. Main result. We are interested in the Beurling operator

$$
T \varphi(z):=\frac{1}{\pi} \iint \frac{\varphi(\zeta) d A(\zeta)}{(\zeta-z)^{2}}
$$


understood as a Calderón-Zygmund operator ( $d A$ denotes area Lebesgue measure on $\mathbb{C}$ ). This operator has the important property that it turns $\bar{z}$ derivatives into $z$-derivatives:

$$
B\left(\partial_{\bar{z}} f\right)=\partial_{z} f
$$

Here is our main result:

THEOREM 2.1. The following set of conditions is sufficient for the boundedness of the Beurling operator from $L^{2}\left(w^{-1}\right)$ to $L^{2}(v)$ :

$$
\begin{aligned}
& \sup _{(x, t) \in \mathbb{R}_{+}^{3}} \widetilde{w}(x, t) \widetilde{v}(x, t) \leq C, \\
& \mathcal{G}\left(\left|\partial_{1} \widetilde{v}\right|^{2} \widetilde{w}\right)(x, t) \leq C \widetilde{v}(x, t), \\
& \mathcal{G}\left(\left|\partial_{1} \widetilde{w}\right|^{2} \widetilde{v}\right)(x, t) \leq C \widetilde{w}(x, t), \\
& \mathcal{G}\left(\left|\partial_{1} \widetilde{w}\right|\left|\partial_{1} \widetilde{v}\right|\right)(x, t) \leq C, \\
& \mathcal{G}\left(\frac{\left|\partial_{1} \widetilde{w}\right|\left|\partial_{1} \widetilde{v}\right|}{\widetilde{v}}\right)(x, t) \leq C \widetilde{w}(x, t), \\
& \mathcal{G}\left(\frac{\left|\partial_{1} \widetilde{w}\right|\left|\partial_{1} \widetilde{v}\right|}{\widetilde{w}}\right)(x, t) \leq C \widetilde{v}(x, t) .
\end{aligned}
$$

\section{Proof}

3.1. Useful facts. In this section we will give an overview of some useful background information.

We only need to prove the theorem for non-negative functions, since we can then extend it to all functions by linearity. The heat extension of a non-negative, non-zero function is positive, since the heat kernel is positive. The fundamental solution of the heat equation, $\Phi(x, t)$, is also non-negative, so the Green's potential $\mathcal{G}(f)(x, t)$ of a non-negative function $f(x, t)$ is nonnegative. We can use the Cauchy-Schwarz inequality for heat extensions: $(\widetilde{f g})^{2} \leq \widetilde{f^{2}} \widetilde{g^{2}}$.

The Green's function for the heat equation in the cylinder is a nonnegative function $G$ with the following properties [7, 15]:

$$
\left\{\begin{array}{l}
\left(\frac{\partial}{\partial t}+\Delta\right) G=-\delta_{0,1 / 2} \quad \text { in } C(1,1)=B(0,1) \times(0,1), \\
G=0 \quad \text { on } \partial^{\prime} C(1,1)=\partial B(0,1) \times(0,1) \\
G=0 \quad \text { when } t=1 .
\end{array}\right.
$$

Here $\delta_{0,1 / 2}$ is the Dirac measure at the point $(0,1 / 2)$. Furthermore

$$
G(x, 0) \geq a(1-\|x\|),
$$

where $a$ is a positive absolute constant. 
We will also use Green's function $G_{R}$ adapted to the cylinder $C\left(R, R^{2}\right)$ $=B(0, R) \times\left(0, R^{2}\right)$ :

$$
\left\{\begin{array}{l}
\left(\frac{\partial}{\partial t}+\Delta\right) G^{R}=-\delta_{0, R^{2} / 2} \quad \text { in } C\left(R, R^{2}\right), \\
G^{R}=0 \quad \text { on } \partial^{\prime} C\left(R, R^{2}\right)=\partial B(0, R) \times\left(0, R^{2}\right), \\
G^{R}=0 \quad \text { when } t=R^{2} .
\end{array}\right.
$$

One can easily see that the following connection holds:

$$
G^{R}(x, t)=\frac{1}{R^{2}} G\left(\frac{x}{R}, \frac{t}{R^{2}}\right) .
$$

Let $b$ be a positive, sufficiently differentiable function. Then we have the following Green's formula, which is a consequence of integration by parts and properties of $G[15]$ :

$$
\begin{aligned}
b\left(0, R^{2} / 2\right) & =-\iiint_{C\left(R, R^{2}\right)}\left(\frac{\partial}{\partial t}+\Delta\right) G^{R}(x, t) b(x, t) d x_{1} d x_{2} d t \\
& \geq \iiint_{C\left(R, R^{2}\right)} G^{R}(x, t)\left(\frac{\partial}{\partial t}-\Delta\right) b(x, t) d x_{1} d x_{2} d t .
\end{aligned}
$$

Given a smooth function $B$ and a vector-function $k(x, t)$ whose entries are solutions to the heat equation or Green's potential functions, we will have to relate $\left(\partial_{t}-\Delta\right) B(k(x, t))$ to the Hessian of $B$. The following lemma is proven by straightforward differentiation.

Lemma 3.1 ([15]). Let

$$
k(x, t)=\left(f_{1}(x, t), \ldots, f_{s}(x, t), \mathcal{G}\left(g_{1}\right)(x, t), \ldots, \mathcal{G}\left(g_{m}\right)(x, t)\right),
$$

where the $f_{i}(x, t)$ are the heat extensions of $f_{i}(x)$, and the $\mathcal{G}\left(g_{i}\right)$ are Green's potentials of $g_{i}$. Then for $B$ a function of $s+m$ variables and $b(x, t)=$ $B(k(x, t))$,

$$
\begin{aligned}
& \left(\frac{\partial}{\partial t}-\Delta\right) b(x, t) \\
& =\left(\left(-d^{2} B\right) \frac{\partial k}{\partial x_{1}}, \frac{\partial k}{\partial x_{1}}\right)_{\mathbb{R}^{s+m}}+\left(\left(-d^{2} B\right) \frac{\partial k}{\partial x_{2}}, \frac{\partial k}{\partial x_{2}}\right)_{\mathbb{R}^{s+m}}+\sum_{i=1}^{m} \frac{\partial B}{\partial k_{s+i}} g_{i}(x, t) .
\end{aligned}
$$

3.2. Transforming the estimate. In this section we reduce the required estimate to an estimate on four integrals that do not directly involve the Beurling operator itself anymore. We will use the following fact from [15] which we state here as a lemma:

LEMMA 3.2 .

$$
\left\|R_{i}^{2} f\right\|_{v} \leq C\|f\|_{w^{-1}}, i=1,2 \Rightarrow\|T f\|_{v} \leq C\|f\|_{w^{-1}} .
$$


In other words, to estimate the Beurling operator, it is sufficient to estimate the squares of the Riesz transforms. This lemma follows from the fact that the Fourier multiplier for the Beurling operator is a combination of the squares of the multipliers of the Riesz transforms and rotations thereof. In what follows, we will estimate $R_{1}^{2}$; the proof for $R_{2}^{2}$ is analogous.

By $L^{2}$ duality it is enough to show the following inequality for test functions:

$$
\left|\left(R_{1}^{2} \phi, \psi\right)\right| \leq C\|\phi\|_{w^{-1}}\|\psi\|_{v^{-1}} .
$$

This follows if we prove

$$
\left|\left(R_{1}^{2} \phi, \psi\right)\right| \leq C\left(\|\phi\|_{w^{-1}}^{2}+\|\psi\|_{v^{-1}}^{2}\right),
$$

since the latter implies that

$$
\left|\left(R_{1}^{2} \phi, \psi\right)\right|=\left|\left(c R_{1}^{2} \phi, \frac{1}{c} \psi\right)\right| \leq C\left(c\|\phi\|_{w^{-1}}^{2}+\frac{1}{c}\|\psi\|_{v^{-1}}^{2}\right) \quad \forall c>0 .
$$

Letting $c=\|\psi\|_{v^{-1}} /\|\phi\|_{w^{-1}}$ brings us back to the original equation.

Instead of estimating the inner product $\left|\left(R^{2} \phi, \psi\right)\right|$ we wish to estimate an integral that involves derivatives of the heat extensions of $\phi$ and $\psi$ instead. To accomplish this, we will use the following lemma from [15] in a crucial way. For completeness, we repeat the proof here.

Lemma 3.3. Let $\varphi, \psi \in C_{0}^{\infty}$. The integral $\iiint \frac{\partial \varphi}{\partial x_{1}} \cdot \frac{\partial \psi}{\partial x_{1}} d x_{1} d x_{2} d t$ converges absolutely and

$$
\iint R_{1}^{2} \varphi \cdot \psi d x_{1} d x_{2}=-2 \iiint_{\mathbb{R}_{+}^{3}} \partial_{1} \widetilde{\phi} \partial_{1} \widetilde{\psi} d x_{1} d x_{2} d t
$$

Proof. We have

$$
\begin{aligned}
\iint \psi R_{1}^{2} \varphi & d x_{1} d x_{2}=\iint \frac{\xi_{1}^{2}}{\xi_{1}^{2}+\xi_{2}^{2}} \widehat{\varphi}\left(\xi_{1}, \xi_{2}\right) \widehat{\psi}\left(-\xi_{1},-\xi_{2}\right) d \xi_{1} d \xi_{2} \\
= & 2 \iiint_{0}^{\infty} e^{-2 t\left(\xi_{1}^{2}+\xi_{2}^{2}\right)} \xi_{1}^{2} \widehat{\varphi}\left(\xi_{1}, \xi_{2}\right) \widehat{\psi}\left(\xi_{1}, \xi_{2}\right) d \xi_{1} d \xi_{2} d t \\
= & -2 \int_{0}^{\infty} \iint i \xi_{1} \widehat{\varphi}\left(\xi_{1}, \xi_{2}\right) e^{-t\left(\xi_{1}^{2}+\xi_{2}^{2}\right)} \cdot i \xi_{1} \widehat{\psi}\left(-\xi_{1},-\xi_{2}\right) e^{-t\left(\xi_{1}^{2}+\xi_{2}^{2}\right)} d \xi_{1} d \xi_{2} d t \\
= & -2 \int_{0}^{\infty} \iint \frac{\partial \varphi}{\partial x_{1}}\left(x_{1}, x_{2}, t\right) \frac{\partial \psi}{\partial x_{1}}\left(x_{1}, x_{2}, t\right) d x_{1} d x_{2} d t \\
= & -2 \iiint_{\mathbb{R}_{+}^{3}} \frac{\partial \varphi}{\partial x_{1}}\left(x_{1}, x_{2}, t\right) \frac{\partial \psi}{\partial x_{1}}\left(x_{1}, x_{2}, t\right) d x_{1} d x_{2} d t .
\end{aligned}
$$

Here we have used Parseval's formula twice, as well as the absolute conver- 
gence of the two integrals $\iiint_{\mathbb{R}_{+}^{3}} e^{-2 t\left(\xi_{1}^{2}+\xi_{2}^{2}\right)} \xi_{1}^{2} \widehat{\varphi}\left(\xi_{1}, \xi_{2}\right) \widehat{\psi}\left(\xi_{1}, \xi_{2}\right) d \xi_{1} d \xi_{2} d t$ and $\iiint_{\mathbb{R}_{+}^{3}} \frac{\partial \varphi}{\partial x_{1}}\left(x_{1}, x_{2}, t\right) \frac{\partial \psi}{\partial x_{1}}\left(x_{1}, x_{2}, t\right) d x_{1} d x_{2} d t$. For the first integral this is obvious. The absolute convergence of the second integral can be quite easily proved.

By Lemmas 3.2 and 3.3 we see that it suffices to estimate

$$
\iiint_{\mathbb{R}_{+}^{3}}\left|\partial_{1} \widetilde{\phi} \partial_{1} \widetilde{\psi}\right| d x_{1} d x_{2} d t .
$$

Now we strategically add and subtract terms in the integral, split it, and estimate each resulting integral separately:

$$
\begin{aligned}
\iiint_{\mathbb{R}_{+}^{3}}\left|\partial_{1} \widetilde{\phi} \partial_{1} \widetilde{\psi}\right| d x_{1} d x_{2} d t \leq & \iiint_{\mathbb{R}_{+}^{3}}|\widetilde{\phi}||\widetilde{\psi}|\left|\frac{\partial_{1} \widetilde{\phi}}{\widetilde{\phi}}-\frac{\partial_{1} \widetilde{w}}{\widetilde{w}}\right|\left|\frac{\partial_{1} \widetilde{\psi}}{\widetilde{\psi}}-\frac{\partial_{1} \widetilde{v}}{\widetilde{v}}\right| d A(x) d t \\
& +\iiint_{\mathbb{R}_{+}^{3}}|\widetilde{\phi}||\widetilde{\psi}|\left|\frac{\partial_{1} \widetilde{w}}{\widetilde{w}}\right|\left|\frac{\partial_{1} \widetilde{\psi}}{\widetilde{\psi}}-\frac{\partial_{1} \widetilde{v}}{\widetilde{v}}\right| d A(x) d t \\
& +\iiint_{\mathbb{R}_{+}^{3}}|\widetilde{\phi}||\widetilde{\psi}|\left|\frac{\partial_{1} \widetilde{\phi}}{\widetilde{\phi}}-\frac{\partial_{1} \widetilde{w}}{\widetilde{w}}\right|\left|\frac{\partial_{1} \widetilde{v}}{\widetilde{v}}\right| d A(x) d t \\
& +\iiint_{\mathbb{R}_{+}^{3}}|\widetilde{\phi}||\widetilde{\psi}|\left|\frac{\partial_{1} \widetilde{w}}{\widetilde{w}}\right|\left|\frac{\partial_{1} \widetilde{v}}{\widetilde{v}}\right| d A(x) d t \\
= & I_{1}+I_{2}+I_{3}+I_{4} .
\end{aligned}
$$

We turn to estimating the integrals $I_{1}-I_{4}$.

3.3. Estimate of $I_{1}$. Starting with the easiest estimate, we need to bound

$$
I_{1}=\iiint_{\mathbb{R}_{+}^{3}}|\widetilde{\phi}||\widetilde{\psi}|\left|\frac{\partial_{1} \widetilde{\phi}}{\widetilde{\phi}}-\frac{\partial_{1} \widetilde{w}}{\widetilde{w}}\right|\left|\frac{\partial_{1} \widetilde{\psi}}{\widetilde{\psi}}-\frac{\partial_{1} \widetilde{v}}{\widetilde{v}}\right| d A(x) d t,
$$

for which we invoke our first Bellman function.

Consider

$$
B(A, a, w, Y, y, v)=A-\frac{a^{2}}{w}+Y-\frac{y^{2}}{v} .
$$

On the domain $A, a, w, Y, y, v \geq 0, a^{2} / w \leq A, y^{2} / v \leq Y$, this function has the following properties, which can be verified by direct calculation:

(i) $0 \leq B(A, a, w, Y, y, v) \leq A+Y$,

(ii) $-d^{2} B=\frac{2 a^{2}}{w}\left|\frac{d a}{a}-\frac{d w}{w}\right|^{2}+\frac{2 y^{2}}{v}\left|\frac{d y}{y}-\frac{d v}{v}\right|^{2}$. 
REMARK 3.4. The notation in the second property, which is very convenient in this context, is to be understood in the following way:

$(d A, d a, d w, d Y, d y, d v)\left(-d^{2} B\right)(d A, d a, d w, d Y, d y, d v)^{t}$

$$
=\frac{2 a^{2}}{w}\left|\frac{d a}{a}-\frac{d w}{w}\right|^{2}+\frac{2 y^{2}}{v}\left|\frac{d y}{y}-\frac{d v}{v}\right|^{2} .
$$

Note that

$$
k(x, t)=\left(\widetilde{\phi^{2} w^{-1}}(x, t), \widetilde{\phi}(x, t), \widetilde{w}(x, t), \widetilde{\psi^{2} v^{-1}}(x, t), \widetilde{\psi}(x, t), \widetilde{v}(x, t)\right)
$$

is in the above domain for every $(x, t)$, by our comments earlier in Section 3.1. Consider

$$
b(x, t)=B(k(x, t))
$$

By Lemma 3.1, we have

$$
\begin{aligned}
\left(\frac{\partial}{\partial t}-\Delta\right) b( & x, t) \\
= & \left(\left(-d^{2} B\right) \frac{\partial k}{\partial x_{1}}, \frac{\partial k}{\partial x_{1}}\right)_{\mathbb{R}^{k+m}}+\left(\left(-d^{2} B\right) \frac{\partial k}{\partial x_{2}}, \frac{\partial k}{\partial x_{2}}\right)_{\mathbb{R}^{k+m}} \\
= & 2 \frac{\widetilde{\phi}^{2}}{\widetilde{w}}\left|\frac{\partial_{1} \widetilde{\phi}}{\widetilde{\phi}}-\frac{\partial_{1} \widetilde{w}}{\widetilde{w}}\right|^{2}+2 \frac{\widetilde{\psi}^{2}}{\widetilde{v}}\left|\frac{\partial_{1} \widetilde{\psi}}{\widetilde{\psi}}-\frac{\partial_{1} \widetilde{v}}{\widetilde{v}}\right|^{2}
\end{aligned}
$$

by (ii). By the Cauchy-Schwarz inequality and the fact that $\widetilde{w} \widetilde{v} \leq c$, this is

$$
\geq C|\widetilde{\phi}||\widetilde{\psi}|\left|\frac{\partial_{1} \widetilde{\phi}}{\widetilde{\phi}}-\frac{\partial_{1} \widetilde{w}}{\widetilde{w}}\right|\left|\frac{\partial_{1} \widetilde{\psi}}{\widetilde{\psi}}-\frac{\partial_{1} \widetilde{v}}{\widetilde{v}}\right| .
$$

Furthermore, by (i),

$$
\begin{aligned}
b\left(0, R^{2} / 2\right) \leq & \widetilde{\phi^{2} w^{-1}}\left(0, R^{2} / 2\right)+\widetilde{\psi^{2} v^{-1}}\left(0, R^{2} / 2\right) \\
& =\frac{1}{\pi} \frac{2}{R^{2}} \iint_{\mathbb{R}^{2}}\left(\phi^{2} w^{-1}(x)+\psi^{2} v^{-1}(x)\right) \exp \left(\frac{-2|x|^{2}}{4 R^{2}}\right) d A(x) \\
& \leq \frac{2}{\pi R^{2}}\left(\|\phi\|_{w^{-1}}^{2}+\|\psi\|_{v^{-1}}^{2}\right) .
\end{aligned}
$$

Putting these together, and using our Green's formula (3.2), we get

$$
\begin{aligned}
\frac{2}{\pi R^{2}} & \left(\|\phi\|_{w^{-1}}^{2}+\|\psi\|_{v^{-1}}^{2}\right) \geq b\left(0, R^{2} / 2\right) \\
& \geq \iiint_{C\left(R, R^{2}\right)} G^{R}(x, t)\left(\frac{\partial}{\partial t}-\Delta\right) b(x, t) d x_{1} d x_{2} d t \\
& \geq \frac{C}{R^{2}} \iiint_{C\left(R, R^{2}\right)} G^{1}\left(\frac{x}{R}, \frac{t}{R^{2}}\right)|\widetilde{\phi}||\widetilde{\psi}|\left|\frac{\partial_{1} \widetilde{\phi}}{\widetilde{\phi}}-\frac{\partial_{1} \widetilde{w}}{\widetilde{w}}\right|\left|\frac{\partial_{1} \widetilde{\psi}}{\widetilde{\psi}}-\frac{\partial_{1} \widetilde{v}}{\widetilde{v}}\right| d x_{1} d x_{2} d t .
\end{aligned}
$$


Multiply both sides by $R^{2}$ and let $R \rightarrow \infty$ (keeping in mind that $G^{1}(0,0)$ $>0)$ to obtain

$$
\frac{2}{\pi}\left(\|\phi\|_{w^{-1}}^{2}+\|\psi\|_{v^{-1}}^{2}\right) \geq C \iiint_{\mathbb{R}_{+}^{3}}|\widetilde{\phi}||\widetilde{\psi}|\left|\frac{\partial_{1} \widetilde{\phi}}{\widetilde{\phi}}-\frac{\partial_{1} \widetilde{w}}{\widetilde{w}}\right|\left|\frac{\partial_{1} \widetilde{\psi}}{\widetilde{\psi}}-\frac{\partial_{1} \widetilde{v}}{\widetilde{v}}\right| d A(x) d t
$$

as desired.

3.4. Estimates of $I_{2}, I_{3}$. We will prove the bound of the second integral. The third is handled in the same manner.

Thus we wish to bound

$$
I_{2}=\iiint_{\mathbb{R}_{+}^{3}}|\widetilde{\phi}||\widetilde{\psi}| \frac{\partial_{1} \widetilde{w}}{\widetilde{w}}|| \frac{\partial_{1} \widetilde{\psi}}{\widetilde{\psi}}-\frac{\partial_{1} \widetilde{v}}{\widetilde{v}} \mid d A(x) d t .
$$

Consider

$B(A, a, w, Y, y, v, M)=A-\frac{a^{2}}{w+M}+Y-\frac{y^{2}}{v}=B_{1}(A, a, w, M)+B_{2}(Y, y, v)$.

In the domain $A, a, w, Y, y, v \geq 0,0<M \leq C w, a^{2} / w \leq A, y^{2} / v \leq Y$ this function satisfies

(i) $0 \leq B(A, a, w, Y, y, v, M) \leq A+Y$,

(ii) $-d^{2} B_{1} \geq 0$,

(iii) $\partial_{M} B_{1} \geq \frac{a^{2}}{C w^{2}}$,

(iv) $-d^{2} B_{2}=\frac{2 y^{2}}{v}\left|\frac{d y}{y}-\frac{d v}{v}\right|^{2}$.

Note that $k(x, t)=\left(\widetilde{\phi^{2} w^{-1}}, \widetilde{\phi}, \widetilde{w}, \widetilde{\psi^{2} v^{-1}}, \widetilde{\psi}, \widetilde{v}, \mathcal{G}\left(\left(\partial_{1} \widetilde{w}\right)^{2} \widetilde{v}\right)\right)(x, t)$ is in the above domain for every $(x, t)$. In particular, we use here assumption (2.3),

$$
\mathcal{G}\left(\left|\partial_{1} \widetilde{w}\right|^{2} \widetilde{v}\right)(x, t) \leq C \widetilde{w}(x, t)
$$

for the estimate on the derivative in $M$. Now consider

$$
b(x, t)=B(k(x, t)) .
$$

By Lemma 3.1,

$$
\begin{aligned}
& \left(\frac{\partial}{\partial t}-\Delta\right) b(x, t) \geq 2 \frac{\widetilde{\psi}^{2}}{\widetilde{v}}\left|\frac{\partial_{1} \widetilde{\psi}}{\widetilde{\psi}}-\frac{\partial_{1} \widetilde{v}}{\widetilde{v}}\right|^{2}+\frac{\partial B}{\partial M}\left(\partial_{1} \widetilde{w}\right)^{2} \widetilde{v} \\
& \geq 2 \frac{\widetilde{\psi}^{2}}{\widetilde{v}}\left|\frac{\partial_{1} \widetilde{\psi}}{\widetilde{\psi}}-\frac{\partial_{1} \widetilde{v}}{\widetilde{v}}\right|^{2}+\frac{\widetilde{\phi}^{2}}{C \widetilde{w}^{2}}\left(\partial_{1} \widetilde{w}\right)^{2} \widetilde{v} \geq C \frac{\widetilde{\psi}}{\widetilde{v}}\left|\frac{\partial_{1} \widetilde{\psi}}{\widetilde{\psi}}-\frac{\partial_{1} \widetilde{v}}{\widetilde{v}}\right| \frac{\widetilde{\phi}}{\widetilde{w}}\left(\partial_{1} \widetilde{w}\right) \widetilde{v}
\end{aligned}
$$

As before,

$$
b\left(0, R^{2} / 2\right) \leq \frac{2}{\pi R^{2}}\left(\|\phi\|_{w^{-1}}^{2}+\|\psi\|_{v^{-1}}^{2}\right) .
$$


Combining the estimates gives

$$
\begin{aligned}
& \frac{2}{\pi R^{2}}\left(\|\phi\|_{w^{-1}}^{2}+\|\psi\|_{v^{-1}}^{2}\right) \geq b\left(0, R^{2} / 2\right) \\
& \quad \geq \iiint_{C\left(R, R^{2}\right)} G^{R}(x, t)\left(\frac{\partial}{\partial t}-\Delta\right) b(x, t) d x_{1} d x_{2} d t \\
& \quad \geq \frac{C}{R^{2}} \iiint \int_{C\left(R, R^{2}\right) \cap\{t>\varepsilon\}} G^{1}\left(\frac{x}{R}, \frac{t}{R^{2}}\right) \widetilde{\psi} \widetilde{\phi} \frac{\partial_{1} \widetilde{w}}{\widetilde{w}}\left|\frac{\partial_{1} \widetilde{\psi}}{\widetilde{\psi}}-\frac{\partial_{1} \widetilde{v}}{\widetilde{v}}\right| d x_{1} d x_{2} d t .
\end{aligned}
$$

As before, multiply by $R^{2}$, and let $R \rightarrow \infty$.

3.5. Estimate of $I_{4}$. This integral is the most difficult to estimate and a careful analysis of the corresponding dyadic situation was necessary to establish the Bellman functions considered. These go back to [11], [19] and [16]. We wish to prove that

$$
\iiint_{\mathbb{R}_{+}^{3}}|\widetilde{\phi}||\widetilde{\psi}|\left|\frac{\partial_{1} \widetilde{w}}{\widetilde{w}}\right|\left|\frac{\partial_{1} \widetilde{v}}{\widetilde{v}}\right| d A(x) d t \leq C\left(\|\phi\|_{w^{-1}}^{2}+\|\psi\|_{v^{-1}}^{2}\right) .
$$

We will do this via the following Bilinear Embedding Theorem (see different versions also in $[11,16])$ :

THEOREM 3.5. If $\alpha(x, t) \geq 0$ and $w, v$ are two weights so that $0<$ $\widetilde{w}(x, t) \widetilde{v}(x, t) \leq C$ for all $(x, t) \in \mathbb{R}_{+}^{3}$ and

$$
\begin{aligned}
& \mathcal{G}(\alpha(x, t) \widetilde{w}(x, t) \widetilde{v}(x, t)) \leq C, \\
& \mathcal{G}(\alpha(x, t) \widetilde{w}(x, t)) \leq C \widetilde{w}(x, t), \\
& \mathcal{G}(\alpha(x, t) \widetilde{v}(x, t)) \leq C \widetilde{v}(x, t),
\end{aligned}
$$

then for $\phi, \psi \geq 0$ in $L^{2}$ we have

$$
\iiint_{\mathbb{R}_{+}^{3}} \alpha(x, t) \widetilde{\phi}(x, t) \widetilde{\psi}(x, t) d A(x) d t \leq C^{\prime}\left(\|\phi\|_{w^{-1}}^{2}+\|\psi\|_{v^{-1}}^{2}\right) .
$$

Thus, if we let

$$
\alpha(x, t)=\frac{\left|\partial_{1} \widetilde{w}(x, t)\right|\left|\partial_{1} \widetilde{v}(x, t)\right|}{\widetilde{w}(x, t) \widetilde{v}(x, t)},
$$

and use Theorem 3.5 and assumptions (2.4)-(2.6), then $I_{4}$ is bounded as desired.

It remains to prove Theorem 3.5.

3.5.1. Proof of the Bilinear Embedding Theorem. The Bellman function in this section will be complicated, and its properties non-trivial. 
Consider the following set of variables, and the following domain:

$$
\begin{aligned}
& \{(A, a, r, Y, y, s, M, N, K): A, a, r, Y, y, s, M, N, K>0 ; \\
& \left.0 \leq r s \leq C ; a^{2} \leq A r ; y^{2} \leq Y s ; M \leq C r ; N \leq C s ; K \leq C\right\} .
\end{aligned}
$$

Note that this domain requires that $0 \leq r s \leq C$, as opposed to $1 \leq r s \leq C$ in [16].

Now consider the following Bellman function from [16]:

$$
\begin{aligned}
& B(A, a, r, Y, y, s, M, N, K) \\
& \quad=B_{1}(A, a, r, M)+B_{2}(Y, y, s, N)+B_{3}(A, a, r, Y, y, s, K),
\end{aligned}
$$

where

$$
\begin{gathered}
B_{1}(A, a, r, M)=A-\frac{a^{2}}{r+M}, \\
B_{2}(Y, y, s, N)=Y-\frac{y^{2}}{s+N}, \\
B_{3}(A, a, r, Y, y, s, K) \\
= \begin{cases}A+Y-\frac{a^{2} s-2 a y K+y^{2} r}{r s-K^{2}} & \text { if } y r-a K>0, a s-y K>0, \\
A+Y-y^{2} / s & \text { otherwise, and } a^{2} / r \geq y^{2} / s, \\
A+Y-a^{2} / r & \text { otherwise, and } a^{2} / r \leq y^{2} / s .\end{cases}
\end{gathered}
$$

Lemma 3.6. This Bellman function satisfies the following estimates:

(i) $\frac{\partial B_{1}}{\partial M} \geq C \frac{a^{2}}{r^{2}}$,

$$
\begin{aligned}
& \frac{\partial B_{2}}{\partial N} \geq C \frac{y^{2}}{s^{2}}, \\
& \frac{\partial B_{3}}{\partial K} \geq \begin{cases}c \frac{a y}{r s} & \text { if both } K \leq \frac{y r}{4 a} \text { and } K \leq \frac{a s}{4 y}, \\
0 & \text { else, }\end{cases}
\end{aligned}
$$

(ii) $B$ is concave,

(iii) $0 \leq B \leq 3(A+Y)$.

Proof. The first two derivative conditions are straightforward. As for the third, a calculation shows that

$$
\frac{\partial B_{3}}{\partial K}=\frac{2(a s-y K)(y r-a K)}{\left(K^{2}-r s\right)^{2}}
$$

for $y r>a K$ and $a s>y K$, and $\partial B_{3} / \partial K=0$ else. That proves nonnegativity.

Furthermore, when $a K \leq y r / 4$ and $y K \leq a s / 4$, we have $y r-a K \geq$ $3 / 4 y r$, as $-y K \geq a s-a s / 4=3 a s / 4$ and $K^{2} a y \leq y r a s / 16$. The latter implies that $K^{2} \leq r s / 16$, so $0<r s-K^{2} \leq r s$. 
Thus in that range

$$
\frac{2(a s-y K)(y r-a K)}{\left(K^{2}-r s\right)^{2}} \geq C \frac{a s y r}{\left(K^{2}-r s\right)^{2}} \geq C \frac{a y}{r s},
$$

which proves the third derivative estimate.

It is easy to check that the matrices $-d^{2} B_{1},-d^{2} B_{2}$ are positive semidefinite, and thus $B_{1}$ and $B_{2}$ are concave. As for $B_{3}$, it is useful here to observe that

$$
B_{3}=A+Y-\sup _{\alpha>0}\left(\frac{a^{2}}{r+\alpha K}+\frac{y^{2}}{s+\alpha^{-1} K}\right)
$$

(see $[11,16])$. The quantity in parentheses is convex. Thus the function as a whole is an infimum of concave functions, and therefore concave. Hence $B$ is concave.

Furthermore, $0 \leq B_{i} \leq A+Y$ for $i=1,2,3$. The upper bounds are obvious, as is the positivity, except in the case of $B_{3}$ when $y r-A K>0$ and as $-y K>0$. For that case, note that

$$
A+Y-\frac{a^{2} s-2 a y K+y^{2} r}{r s-K^{2}}=A-\frac{a(a s-y K)}{r s-K^{2}}+Y-\frac{y(y r-a K)}{r s-K^{2}} .
$$

We will prove that

$$
A \geq \frac{a(a s-y K)}{r s-K^{2}} .
$$

The other half goes the same way.

Since as $>y K$, we see that $\alpha_{1}=a s / y K>1$, and similarly $\alpha_{2}=$ $y r / a K>1$, and

$$
\frac{a s y r}{y K a K}=\frac{s r}{K^{2}}=\alpha_{1} \alpha_{2}>1
$$

Thus

$$
\frac{a(a s-y K)}{r s-K^{2}}=\frac{a\left(1-\frac{1}{\alpha_{1}}\right) a s}{\left(1-\frac{1}{\alpha_{1} \alpha_{2}}\right) s r}=\frac{1-\frac{1}{\alpha_{1}}}{1-\frac{1}{\alpha_{1} \alpha_{2}}} \frac{a^{2}}{r} \leq A,
$$

since $0<\frac{1-1 / \alpha_{1}}{1-1 / \alpha_{1} \alpha_{2}}=\frac{\alpha_{1} \alpha_{2}-\alpha_{2}}{\alpha_{1} \alpha_{2}-1}=1+\frac{1-\alpha_{2}}{\alpha_{1} \alpha_{2}-1} \leq 1$.

As before, we wish to plug in heat extensions and Green's potentials for our variables.

We let

$$
\begin{gathered}
A=\widetilde{\phi^{2} w^{-1}}, \quad a=\widetilde{\phi}, \quad r=\widetilde{w}, \\
Y=\widetilde{\psi^{2} v^{-1}}, \quad y=\widetilde{\psi}, \quad s=\widetilde{v}, \\
K=\mathcal{G}(\alpha \widetilde{w} \widetilde{v}), \quad M=\mathcal{G}(\alpha \widetilde{v} K), \quad N=\mathcal{G}(\alpha \widetilde{w} K) .
\end{gathered}
$$

All of these variables are positive. Moreover, the assumptions of the lemma imply that 


$$
K \leq C, \quad M=\mathcal{G}(\alpha \widetilde{v} K) \leq C \mathcal{G}(\alpha \widetilde{v}) \leq C^{\prime} \widetilde{v}(x, t), \quad N \leq C^{\prime} \widetilde{w}(x, t) .
$$

Thus $k(x, t)=\left(\widetilde{\phi^{2} w^{-1}}, \widetilde{\phi}, \widetilde{w}, \widetilde{\psi^{2} v^{-1}}, \widetilde{\psi}, \widetilde{v}, \mathcal{G}(\alpha \widetilde{v}(\mathcal{G}(\alpha \widetilde{w} \widetilde{v}))), \mathcal{G}(\alpha \widetilde{w}(\mathcal{G}(\alpha \widetilde{w} \widetilde{v})))\right.$, $\mathcal{G}(\alpha \widetilde{w} \widetilde{v}))$ is in the domain of $B$.

We wish to use $b(x, t)=B(k(x, t))$. In this case, however, $B$ (or more specifically $B_{3}$ ) is not smooth enough to use in the Green's formula. Thus we will mollify $B_{3}$ first.

For $\delta$ small, we consider the set $D_{\delta}=\left\{k(x, t):(x, t) \in \bar{C}^{\delta}\left(R, R^{2}\right)\right\}$. Since $\bar{C}^{\delta}\left(R, R^{2}\right)$ is compact, $D_{\delta}$ is a compact subset of the domain of $B$. Let $\varepsilon(\delta)$ be the smallest distance between $D_{\delta}$ and the union of the hyperplanes $A=0, a=0, r=0, Y=0, y=0, s=0, M=0, N=0, K=0$. We pick $\phi_{\varepsilon(\delta)}$ to be a $C^{\infty}$ approximate identity in $\mathbb{R}^{9}$ with radius $\varepsilon(\delta) / 2$. By this we mean a smooth, radial and non-negative bump function supported by a disk around 0 of radius $\varepsilon(\delta) / 2$ and normalized to $\int \phi_{\varepsilon(\delta)}=1$. We consider the convolution $B_{3}^{\delta}=B_{3} * \phi_{\varepsilon(\delta)}$. The resulting function is smooth in $D_{\delta}$ and it has size, derivative and concavity properties similar to $B_{3}$. It is easy to see that only the constants change by a factor of $3 / 2$ for the size estimate and by $1 / 9$ in the case of the derivative estimate for all choices of $\delta$. The derivative estimate of $B_{3}^{\delta}$ will only hold for smaller $K$, namely $\{K \leq y r / 18 a$ and $K \leq a s / 18 y\}$. In this sense

$$
-d^{2} B_{3}^{\delta} \geq 0 \quad \text { in } D_{\delta}
$$

Let $b_{i}(x, t)=B_{i}(k(x, t))$ for $i=1,2$, and $b_{3}^{\delta}(x, t)=b_{3}^{\delta}(k(x, t))$. The sum $b_{1}+b_{2}+b_{3}^{\delta}$ is denoted by $b^{\delta}$. This function is defined on $\bar{C}^{\delta}\left(R, R^{2}\right)$ only, so we estimate our integral on a slightly smaller region first and then pass to the limit. We divide $\bar{C}^{\delta}\left(R, R^{2}\right)$ into three parts

$$
\begin{aligned}
& A_{1}=\left\{(x, t) \in \bar{C}^{\delta}\left(R, R^{2}\right): K(x, t) \geq \frac{\widetilde{\psi(x, t)} \widetilde{v}(x, t)}{18 \widetilde{\phi}(x, t)}\right\}, \\
& A_{2}=\left\{(x, t) \in \bar{C}^{\delta}\left(R, R^{2}\right): K(x, t) \geq \frac{\widetilde{\phi}(x, t) \widetilde{w}(x, t)}{18 \widetilde{\psi}(x, t)}\right\}, \\
& A_{3}=\bar{C}^{\delta}\left(R, R^{2}\right) \backslash\left(A_{1} \cup A_{2}\right) .
\end{aligned}
$$

If $(x, t) \in A_{1}$, then

$$
\begin{aligned}
\left(\frac{\partial}{\partial t}-\Delta\right) b_{1}(x, t) & \geq \frac{\partial B_{1}}{\partial M}\left(\left(\frac{\partial}{\partial t}-\Delta\right) M\right) \geq \frac{1}{4 Q^{2}} \frac{\widetilde{\phi}(x, t)^{2}}{\widetilde{v}(x, t)^{2}} \alpha(x, t) \widetilde{v}(x, t) K(x, t) \\
& \geq \frac{1}{4 Q^{2}} \frac{\widetilde{\phi}(x, t)^{2}}{\widetilde{v}(x, t)^{2}} \alpha(x, t) \widetilde{v}(x, t) Q \frac{\widetilde{\psi}(x, t) \widetilde{v}(x, t)}{18 \widetilde{\phi}(x, t)} \\
& =\frac{1}{72 Q} \alpha(x, t) \widetilde{\phi}(x, t) \widetilde{\psi}(x, t) .
\end{aligned}
$$


Similarly, if $(x, t) \in A_{2}$, then

$$
\left(\frac{\partial}{\partial t}-\Delta\right) b_{2}(x, t) \geq \frac{1}{72 Q} \alpha(x, t) \widetilde{\phi}(x, t) \widetilde{\psi}(x, t) .
$$

If $(x, t) \in A_{3}$ then

$$
\begin{aligned}
\left(\frac{\partial}{\partial t}-\Delta\right) b_{3}^{\delta}(x, t) & \geq \frac{\partial b_{3}^{\delta}}{\partial K}\left(\left(\frac{\partial}{\partial t}-\Delta\right) K\right) \\
& \geq \frac{c}{Q} \frac{\widetilde{\phi}(x, t) \widetilde{\psi}(x, t)}{\widetilde{w}(x, t) \widetilde{v}(x, t)} \alpha(x, t) \widetilde{w}(x, t) \widetilde{v}(x, t) \\
& =\frac{c}{Q} \alpha(x, t) \widetilde{\phi}(x, t) \widetilde{\psi}(x, t) .
\end{aligned}
$$

Since $(\partial / \partial t-\Delta) b_{1,2},(\partial / \partial t-\Delta) b_{3}^{\delta} \geq 0$ on all of $\bar{C}^{\delta}\left(R, R^{2}\right)$ we have altogether

$$
\left(\frac{\partial}{\partial t}-\Delta\right) b^{\delta}(x, t) \geq \alpha(x, t) \widetilde{\phi}(x, t) \widetilde{\psi}(x, t) .
$$

Now by the Green's formula (3.2),

$$
\begin{aligned}
b^{\delta}\left(0,(R-\delta)^{2} / 2\right) & \geq \iiint_{\bar{C}^{\delta}\left(R, R^{2}\right)} G^{R-\delta}(x, t)\left(\frac{\partial}{\partial t}-\Delta\right) b^{\delta}(x, t) d x d t \\
& \geq \iiint_{\bar{C}^{\delta}\left(R, R^{2}\right)} G^{R-\delta}(x, t) \alpha(x, t) \widetilde{\phi}(x, t) \widetilde{\psi}(x, t) d x d t \\
& =\iiint_{\bar{C}^{\delta}\left(R, R^{2}\right)} \frac{1}{(R-\delta)^{2}} G(0,1) \alpha(x, t) \widetilde{\phi}(x, t) \widetilde{\psi}(x, t) d x d t \\
& \geq \frac{C}{(R-\delta)^{2}} \int_{\bar{C}^{\delta}\left(R, R^{2}\right)} \alpha(x, t) \widetilde{\phi}(x, t) \widetilde{\psi}(x, t) d x d t .
\end{aligned}
$$

By the upper bound on $B$, we see that

$$
\begin{aligned}
b^{\delta}\left(0,(R-\delta)^{2} / 2\right) & \leq \widetilde{\phi^{2} w^{-1}}\left(0,(R-\delta)^{2} / 2\right)+\widetilde{\psi^{2} v^{-1}}\left(0,(R-\delta)^{2} / 2\right) \\
& =\frac{2}{\pi(R-\delta)^{2}} \iint_{\mathbb{R}^{2}} \phi^{2}(y) w^{-1}(y) \exp \left(-\frac{2\|y\|^{2}}{(R-\delta)^{2}}\right) d y \\
& +\frac{2}{\pi(R-\delta)^{2}} \iint_{\mathbb{R}^{2}} \psi^{2}(y) v^{-1}(y) \exp \left(-\frac{2\|y\|^{2}}{(R-\delta)^{2}}\right) d y \\
& \leq \frac{2}{\pi(R-\delta)^{2}}\left(\|\phi\|_{L^{2}\left(w^{-1}\right)}+\|\psi\|_{L^{2}\left(v^{-1}\right)}\right) .
\end{aligned}
$$


Putting these two together, we see that

$$
\begin{aligned}
\frac{C}{(R-\delta)^{2}} \quad \iiint_{C\left((R-\delta),(R-\delta)^{2}\right)} \alpha(x, t) & \widetilde{\phi}(x, t) \widetilde{\psi}(x, t) d x d t \\
& \leq \frac{2}{\pi(R-\delta)^{2}}\left(\|\phi\|_{L^{2}\left(w^{-1}\right)}+\|\psi\|_{L^{2}\left(v^{-1}\right)}\right) .
\end{aligned}
$$

Canceling the $R-\delta$ factors, and letting $R \rightarrow \infty$ and $\delta \rightarrow 0$, we see that

$$
\iiint_{\mathbb{R}_{+}^{3}} \alpha(x, t) \widetilde{\phi}(x, t) \widetilde{\psi}(x, t) d x d t \leq C\left(\|\phi\|_{w^{-1}}^{2}+\|\psi\|_{v^{-1}}^{2}\right),
$$

which finishes the proof of the lemma.

\section{References}

[1] K. Astala, Area distortion of quasiconformal mappings, Acta Math. 173 (1994), $37-60$.

[2] K. Astala and M. J. Gonzalez, Geometry of curves and Beurling operator, preprint.

[3] K. Astala, T. Iwaniec and E. Saksman, Beltrami operators, Duke Math. J. 107 (2001), 27-56.

[4] D. L. Burkholder, Sharp inequalities for martingales and stochastic integrals, in: Colloque Paul Lévy sur les processus stochastiques, Astérisque 157-158 (1988), 75-94.

[5] - A proof of the Petczyński conjecture for the Haar system, Studia Math. 91 (1988), 79-83.

[6] - Differential subordination of harmonic functions and martingales, in: Lecture Notes in Math. 1384, Springer, 1989, 1-23.

[7] R. A. Fefferman, C. E. Kenig and J. Pipher, The theory of weights and the Dirichlet problem for elliptic equations, Ann. of Math. 134 (1991), 65-124.

[8] S. Hukovic, S. Treil and A. Volberg, The Bellman Functions and Sharp Weighted Inequalities for Square Functions, Oper. Theory Adv. Appl. 113, Birkhäuser, 2000.

[9] F. Nazarov and S. Treil, The hunt for a Bellman function: applicatons to estimates for singular integral operators and to other classical problems of harmonic analysis, Algebra i Analiz 8 (1996), no. 5, 32-162 (in Russian); English transl.: St. Petersburg Math. J. 8 (1997), 721-824.

[10] - - - Hunt-Muckenhoupt-Wheeden inequality is now trivial, C. R. Acad. Sci. Paris Sér. I 323 (1996), 717-722.

[11] F. Nazarov, S. Treil and A. Volberg, The Bellman functions and two-weight inequalities for Haar multipliers, J. Amer. Math. Soc. 12 (1999), 909-928.

[12] F. Nazarov and A. Volberg, Heating of the Beurling operator and estimates of its norm, Algebra i Analiz 15 (2003), no. 4, 142-158 (in Russian); English transl.: St. Petersburg Math. J. 15 (2004), 563-573.

[13] - - - The Bellman function, the two-weight Hilbert transform, and embeddings of the model spaces $K_{\theta}$, J. Anal. Math. 87 (2002), 385-414.

[14] S. Petermichl, The sharp bound for the Hilbert transform on weighted Lebesgue spaces in terms of the classical $A_{p}$ characteristic, Amer. J. Math. (2008), to appear. 
[15] S. Petermichl and A. Volberg, Heating of the Ahlfors-Beurling operator: weakly quasiregular maps on the plane are quasiregular, Duke Math. J. 112 (2002), 281-305.

[16] S. Petermichl and J. Wittwer, A sharp weighted estimate on the norm of Hilbert transform via invariant $A_{2}$ characteristic of the weight, Michigan Math. J. 50 (2002), $71-87$.

[17] S. Treil and A. Volberg, Wavelets and the angle between past and future, J. Funct. Anal. 143 (1997), 269-308.

[18] - - - Continuous frame decomposition and matrix Hunt-Muckenhoupt-Wheeden theorem, Ark. Mat. 35 (1997), 363-386.

[19] J. Wittwer, A sharp estimate on the norm of the martingale transform, Math. Res. Lett. 7 (2000), 1-12.

Département de Mathématiques

Westminster College

Université de Bordeaux 1

1840 S 1300 E

351, Cours de la Libération

Salt Lake City, UT 84105, U.S.A.

33405 Talence, France

l: jwittwer@westminstercollege.edu

E-mail: stefanie@math.u-bordeaux1.fr

Received September 23, 2005

Revised version February 28, 2008 